\title{
La investigación como carga académica en la normativa de la UNAH
}

Jorska Pamela Gómez ${ }^{1}$

\section{El concepto de carga académica y sus componentes en la normativa de la UNAH}

La asignación académica, conocida también como carga académica o trabajo académico es una figura jurídica que ha venido siendo construida en la Universidad Nacional Autónoma de Honduras incluso antes de la puesta en marcha de la actual reforma universitaria, que data de 2004, por lo que las construcciones normativas al respecto han rebasado con amplio margen a la realidad práctica durante muchos años.

En el primer Estatuto del Docente Universitario, publicado en $1989^{2}$, la carga académica estaba concebida no sólo en función de las tareas asignadas al profesor, sino como un criterio de valoración del trabajo encargado a las unidades académicas.

Desde entonces ya se le reconocían cinco componentes, entre los cuales estaba la investigación e incluso se mencionaban de manera expresa las actividades que debían integrar el trabajo académico de investigación, por ejemplo: planificación y programación; diseño de proyectos; preparación de insumos para la investigación; realización de actividades de recolección bibliográfica, de observación, medición, experimentación, cuantificación, tabulación e interpretación de datos; diseño de instrumentos de investigación; aplicación de sistemas computacionales a proyectos de investigación; asesoramiento a estudiantes y capacitación en servicio para la investigación.

Posteriormente, entró en vigor el actual Estatuto del Docente Universitario publicado en el año $2000^{3}$ y reformado de manera parcial en el año $2001^{4}$, en el cual no se recogió de manera expresa en el concepto de carga académica la valoración del trabajo encargado a las unidades académicas, ni se retomaron las descripciones de las actividades que debían integrar el componente de investigación.

\footnotetext{
${ }_{1}^{1}$ Profesora de la Dirección de Estudios de Posgrado, Universidad Nacional Autónoma de Honduras: jorska.gomez@gmail.com

${ }^{2}$ Gaceta 25,972 de 31 de octubre de 1989.

${ }^{3}$ Gaceta 29,100 de 17 de febrero de 2000.

${ }^{4}$ Gaceta 29,648 de 4 de diciembre de 2001.
} 
No obstante, en el actual Estatuto del Docente Universitario se conserva una concepción esencialmente integradora de la carga académica, describiéndola como "el conjunto de tareas que en actividades de investigación, docencia, extensión, administración [o gestión académica], orientación o capacitación docente se le asigna a los [profesores] [...] en función de su especialidad y de las necesidades de la institución" (Estatuto del Docente Universitario, 2002, p. 29).

Con base en lo anterior, las opciones de trabajo académico del profesor de la UNAH quedan enmarcadas en los componentes de: investigación, docencia, gestión académica, vinculación universidad-sociedad y orientación y capacitación docente.

Gráfico 1. Opciones para el desarrollo de la carga académica del profesor Universitario

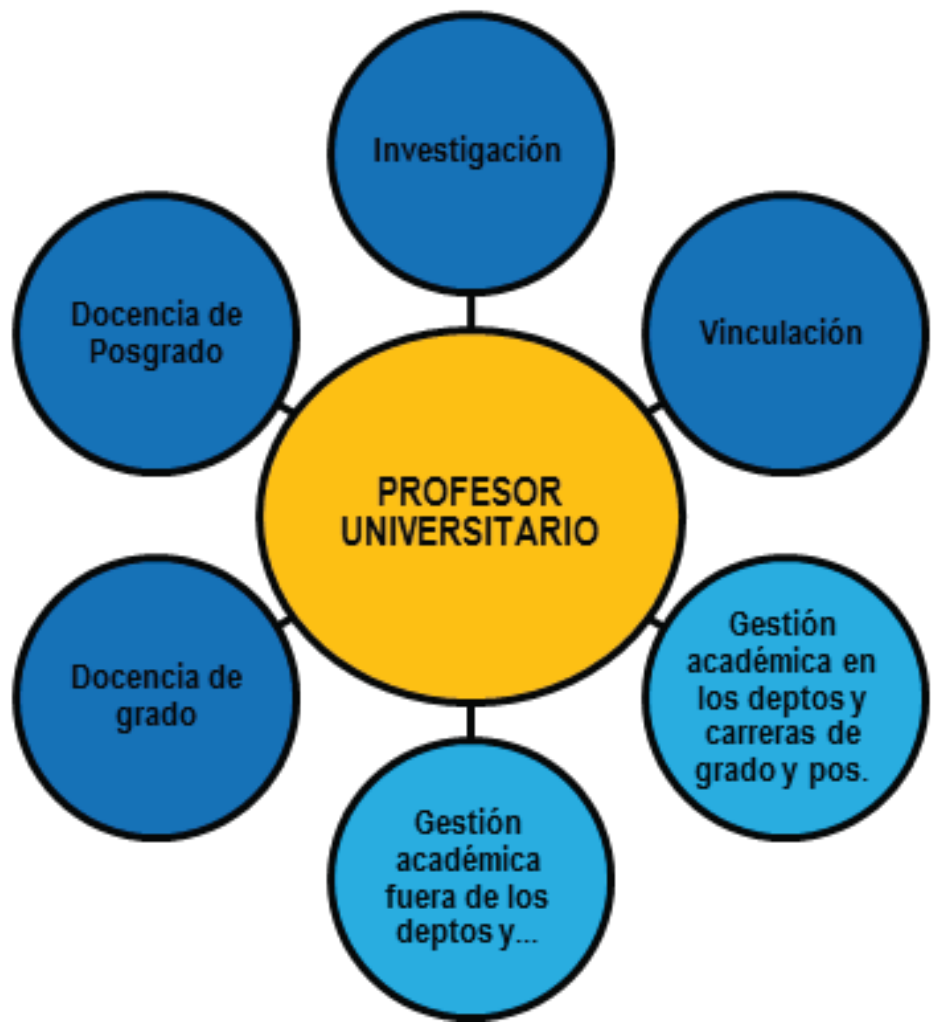

Fuente: Antigua Dirección de Investigación Científica y Posgrado.

La normativa aprobada posteriormente, en el marco de la actual reforma universitaria, recoge este mismo espíritu integrador describiendo el concepto que nos ocupa en los mismos términos. 
Tras la aprobación de las nuevas Normas Académicas de la UNAH, en el año 2015, la figura quedó definitivamente afianzada como un concepto académico, vinculado con las funciones constitucionales encargadas a la universidad y como elemento integral de la carrera docente universitaria ${ }^{5}$.

Cada uno de los componentes de la carga académica hace posible el cumplimiento de alguna de las funciones que la Constitución le otorga a la UNAH, lo que explica por qué fueron incluidos en la descripción de dicho concepto e indica que son fundamentales para comprender el trabajo que lleva a cabo la institución.

Tabla 1. Vínculo de las funciones constitucionales de la UNAH con los componentes de la carga académica

\begin{tabular}{|c|l|}
\hline $\begin{array}{c}\text { Funciones constitucionales de la } \\
\text { UNAH }^{6}\end{array}$ & $\begin{array}{l}\text { Componentes de la carga académica } \\
\text { que contribuyen al logro de la función }\end{array}$ \\
\hline Organizar y dirigir la educación superior & $\begin{array}{l}\text { Gestión académica en los departamentos } \\
\text { y carreras de las facultades } \\
\text { Gestión académica en las direcciones } \\
\text { académicas }\end{array}$ \\
\hline $\begin{array}{c}\text { Desarrollar la educación superior } \\
\text { Orientación y capacitación docente }\end{array}$ & $\begin{array}{l}\text { Docencia en grado, posgrado, educación } \\
\text { presencial y a distancia }\end{array}$ \\
\hline $\begin{array}{c}\text { Contribuir a la investigación científica, } \\
\text { humanística y tecnológica, a la difusión } \\
\text { general de la cultura y al estudio de los } \\
\text { problemas nacionales }\end{array}$ & Investigación (ejecución y gestión) \\
\hline $\begin{array}{c}\text { Participar en la transformación de la } \\
\text { sociedad hondureña }\end{array}$ & Vinculación universidad-sociedad \\
\hline
\end{tabular}

${ }^{5}$ La asignación académica se encuentra descrita en el artículo 277 de las Normas Académicas de la UNAH.

${ }^{6}$ Establecidas en el artículo 160 de la Constitución de la República de Honduras. 
En el componente de investigación de la carga académica la Ley Orgánica reconoce a esta última como un derecho y un deber de los profesores universitarios ${ }^{7}$ y para su impulso promueve como forma de organización administrativa la departamentalización ${ }^{8}$.

Bajo la figura de la departamentalización los profesores universitarios están obligados a desarrollar los componentes de la carga académica en todos los niveles (grado y posgrado) y en todas las modalidades de enseñanza (presencial y a distancia), convirtiéndose en puentes que permiten vincular dichos componentes en una práctica coherente y armónica en estos niveles y modalidades.

También, la figura de la departamentalización pretende garantizar que la organización del trabajo académico responda a las necesidades de las unidades académicas de la institución, ${ }^{9}$ ya que manda que la carga académica de los profesores se programe según los requerimientos de las carreras, institutos y otros programas académicos.

Con esto se espera disminuir la deslegitimación que por muchos años sufrió la investigación como carga académica; para reforzarlo, la Política de Investigación Científica Desarrollo Tecnológico e Innovación (2015-2019) ${ }^{10}$ y el Reglamento del Sistema de Investigación Científica y Tecnológica de la UNAH ${ }^{11}$, aportaron al reconocimiento institucional de la investigación tres elementos más: primero, la expresión de la voluntad de aceptar como tiempo de la jornada de trabajo el dedicado a la formación de competencias metodológicas para la investigación; segundo, disposiciones más específicas para operacionalizar la asignación de investigación como carga académica; y tercero, el mérito académico y profesional del profesor investigador, en términos de categorías, frente a la comunidad universitaria.

Sobre el primer elemento, la política de Investigación Científica Desarrollo Tecnológico e Innovación contempla un componente específico para la capacitación en investigación y en gestión de la investigación y prevé como una de las líneas de acción del

\footnotetext{
7 Artículo 103 Reglamento de la Ley Orgánica de la UNAH, publicado en el diario oficial la Gaceta el 11 de agosto de 2007.

${ }^{8}$ Artículos 92, 93 y 94del Reglamento de la Ley Orgánica de la UNAH, artículo 34 de la Ley Orgánica de la UNAH, artículos 1, 2, 3, 4, 5 y 6 del Reglamento de Departamentos y Carreras de la UNAH. Acuerdo 1947-2013 emitido por la Rectoría el 23 de octubre de 2013.

${ }^{9}$ Artículo 5 del Reglamento de Departamentos y Carreras de la UNAH.

${ }^{10}$ Gaceta 33,958 de 12 de febrero de 2016.

${ }^{11}$ Artículos 12, 13 numeral 3), 28 literal k), 53, 62, 71, 72,73, 74, 75 y 84 literal a) del Reglamento del Sistema de Investigación Científica y Tecnológica de la UNAH.
} 
componente de fomento a la investigación la formación de investigadores en temas prioritarios de investigación. Por lo que el tiempo de participación de los profesores en actividades realizadas en el marco de esta normativa es reconocido institucionalmente dentro de la jornada de trabajo, aunque no sean asignados como parte de la carga académica.

Por otro lado, para el segundo elemento, el Reglamento del Sistema de Investigación Científica y Tecnológica le otorga a los profesores la responsabilidad de realizar la función de investigación encargada a la UNAH, los incorpora como un órgano de la estructura del Sistema de Investigación Científica y Tecnológica y delimita que la carga académica en investigación se considere por un tiempo equivalente a una, dos o tres asignaturas, hasta por tres períodos académicos.

Además, este reglamento legitima el trabajo de investigación realizado por los profesores en los institutos y grupos de investigación, así como en los observatorios y centros experimentales.

Sumado a lo anterior, le confiere a la Dirección de Investigación Científica facultades para evaluar, registrar, dar seguimiento y acreditar la asignación de investigación como carga académica, con lo que se garantiza que las tareas asignadas en este componente realmente sean realizadas, al mismo tiempo que se vigila que cumplan con criterios de calidad.

Finalmente, en cuanto al tercer elemento, este mismo reglamento reconoce cuatro categorías (investigador auxiliar, titular I, titular II y titular III) que ubican a los docentes investigadores según su formación y experiencia en investigación y las cuales deberían impactar en la reclasificación docente establecida en el Estatuto del Docente Universitario.

\section{La ejecución integrada de los componentes investigación y docencia de posgrado en la normativa universitaria}

Las Normas Académicas ${ }^{12}$ y el Modelo Educativo de la UNAH ${ }^{13}$ reconocen a la investigación como una actividad esencial para la construcción del conocimiento, integrándola como eje transversal tanto de los procesos de desarrollo curricular como de los de enseñanza-aprendizaje.

\footnotetext{
${ }^{12}$ Artículo 20 Normas Académicas de la UNAH.

${ }^{13}$ Modelo Educativo de la UNAH página 46.
} 
Lo anterior en concordancia con la perspectiva pedagógica de la UNAH que, según el Reglamento de Departamentos y Carreras ${ }^{14}$, está orientada por la innovación, la creatividad y el cambio, los cuales demandan la práctica de la investigación.

Entonces, para lograr que los fundamentos teóricos que dan sentido al quehacer universitario se ejecuten de manera práctica la Universidad reconoce a los profesores el tiempo invertido en actividades de investigación, a través de la asignación de esta como carga académica.A nivel de posgrado el énfasis en investigación aumenta, en comparación con el grado y se vuelve aún más demandante en los estudios de doctorado que la tienen como base fundamental ${ }^{15}$.

Por tales razones, la UNAH está proyectando que los profesores cuya carga académica abarca el nivel de posgrado sean capaces de combinar los componentes de docencia e investigación y ha incorporado como un mandato a sus Normas Académicas $^{16}$ que todos los departamentos y las carreas de posgrado estén vinculados con una instancia de gestión o ejecución de la investigación científica.

Con ello se asegura la actualización del profesor, el aporte del posgrado a la ciencia, la vinculación de las temáticas desarrolladas con la realidad estudiada a través de las investigaciones y la incorporación de los estudiantes en proyectos dirigidos por los profesores investigadores.

También, se visualiza aumentar la disponibilidad de profesores con competencias para ser asesores temáticos y metodológicos de las tesis, con lo cual se espera impactar positivamente en la eficiencia terminal a nivel de posgrado. Para impulsar esto último, la asesoría de tesis de posgrado puede ser asignada hasta por un tercio de la carga académica de un profesor, lo que le permitiría atender hasta cinco estudiantes durante dos horas al día cada uno, sumando un total de diez horas de asesoría semanales .

\section{La gestión de la investigación como componente de la carga académica}

En el componente de investigación de la carga académica se identifican dos tipos de tareas, las de gestión y las de ejecución directa. Estas tareas forman un todo interrelacionado en el que ambas se aportan insumos. Concentrando la atención en las

\footnotetext{
${ }^{14}$ Artículo 47.

${ }^{15}$ Artículos 39, 39 y 41 del Reglamento General del Sistema de Estudios de Posgrado.

${ }^{16}$ Artículos 23 y 24 de las Normas Académicas de la UNAH.
} 
primeras, encontramos que aporta a las segundas guías de conducción, organización, planificación, integración, seguimiento y evaluación de sus productos, conforme a lo establecido en las Normas Académicas de la UNAH .

Bajo esta lógica, el Reglamento del Sistema de Investigación Científica y Tecnológica de la UNAH reconoce a la Dirección de Investigación Científica, las coordinaciones regionales de investigación científica y las unidades de gestión de la investigación científica como las instancias de gestión de la investigación científica en la UNAH y les otorga unas funciones que están obligadas a cumplir por ley.

Como consecuencia de esto, los profesores que realizan sus labores en las instancias de gestión de la investigación, también, tienen derecho a que su trabajo de gestión sea reconocido como parte de su carga académica.

A pesar de lo anterior, la normativa universitaria contempla muy pocas disposiciones sobre la asignación de gestión en investigación como carga académica, y los casos concretos atendidos por la Dirección de Investigación Científica también son aún reducidos.

En la actualidad, se ha avanzado con un borrador de instructivo para la asignación de gestión en investigación como carga académica, en el cual se describe el máximo de tiempo que un profesor podrá dedicar a esta labor, el procedimiento para la asignación y la forma en que se evaluará el cumplimiento.

No obstante, dicho instructivo aún no se hace efectivo por lo que el reconocimiento de gestión en investigación como carga académica se realiza integrando las labores de gestión con las de ejecución directa en una sola categoría; de modo que a los profesores gestores se les otorga simplemente investigación como carga académica y están sometidos a las mismas reglas de asignación que para la ejecución de la investigación.

\section{CONCLUSIONES}

1. En la normativa que sirve de marco a la actual reforma universitaria la figura de la carga académica, asignación o trabajo académico queda definitivamente afianzada como un concepto académico, vinculado con las funciones constitucionales encargadas a la universidad y elemento integral de la carrera docente universitaria, la 
cual puede ser desarrollada a través de la investigación, docencia, gestión académica, vinculación universidad-sociedad y orientación y capacitación docente.

2. El componente de investigación de la carga académica se concibe como un derecho y un deber; en su faceta de derecho implica el reconocimiento institucional del tiempo dedicado a su gestión y ejecución, además del otorgamiento de méritos tanto académicos como profesionales para el profesor investigador.

Por otro lado, al ser un deber, la distribución del trabajo académico en investigación debe responder a las necesidades de la institución, atender todas las modalidades de enseñanza y enlazarse con la docencia y la vinculación universitaria.

3. De acuerdo con las Normas Académicas todos los departamentos y las carreas de posgrado deben estar vinculados con una instancia de gestión o ejecución de la investigación científica, por lo que la carga académica de los profesores debe incluir tareas de investigación que vinculen a los posgrados con los institutos, grupos, centros experimentales y unidades de gestión de la investigación, así como la asesoría temática y metodológica de las tesis de los estudiantes.

4. La gestión de la investigación científica conforma un todo interrelacionado con las tareas de ejecución directa, de modo que también debe ser reconocida como parte de la carga académica de los profesores universitarios. Sin la gestión de la investigación no sería posible construir conocimiento relevante para guiar la conducción, organización y planificación de la investigación, ni coordinar con mayor agilidad ciertas condiciones materiales imprescindibles para su ejecución.

\section{REFERENCIAS BIBLIOGRÁFICAS}

Acuerdo 1947-2013 emitido por la Rectoría

Constitución de la República de Honduras, Decreto 131 de 1982.

Gaceta 25,972 de 31 de octubre de 1989

Gaceta 29,100 de 17 de febrero de 2000

Gaceta 29,648 de 4 de diciembre de 2001

Ley Orgánica de la UNAH, Decreto 209-2004.

Modelo Educativo de la UNAH (2009). Serie Publicaciones de la Reforma Universitaria No.3. Normas Académicas de la UNAH, Gaceta 33,630 del 13 de enero de 2015.

Política de Investigación Científica Desarrollo Tecnológico e Innovación (2015-2019), Gaceta 33,958 del 12 de febrero de 2016. 
Reglamento de la Ley Orgánica de la UNAH, Acuerdo 206-07.

Reglamento de Departamentos y Carreras de la UNAH, Gaceta 31,545 del 27 de febrero de 2008.

Reglamento del Sistema de Investigación Científica y Tecnológica, Gaceta 33,439 de 29 de mayo de 2014.

Reglamento General del Sistema de Estudios de Posgrado, Gaceta 31,599 del 6 de mayo de 2008. 